\title{
Recubrimientos por PVD decorativos sobre cerámicas
}

\author{
J.A. GARCÍA, A. MARTíNEZ, B. LERGA, M. RICO, G. G. FUENTES, M. ZAZPE Y R.J. RODRÍGUEZ \\ Centro de Ingeniería se Superficies-AIN- Cordovilla 31191 Navarra
}

\begin{abstract}
En los últimos años se ha venido estudiado la aplicabilidad de las técnicas de depósitos mediante métodos físicos en fase vapor (PVD), para la realización de recubrimientos sobre cerámicas y otros substratos con fines decorativos. Los recubrimientos estándar por PVD como el TiN, $\mathrm{CrN}$, TiCN, etc, ofrecen la posibilidad de conseguir acabados de apariencia metálica, de una amplia gama de colores, y con buenas propiedades mecánicas y químicas, como resistencia a la abrasión, estabilidad térmica, o resistencia a la corrosión.

En este trabajo se recogen las experiencias realizadas en el recubrimiento y caracterización de cerámicas decorativas, durante los tres últimos años, en el Centro de Ingeniería de Superficies de la Asociación de la Industria Navarra. Los distintos tipos de recubrimientos se han realizado mediante un equipo de PVD modelo METAPLAS 232, con seis evaporadores de arco eléctrico y sistema de limpieza iónica patentado AEGD. Los recubrimientos han sido caracterizados, tanto química como mecánicamente, empleando las más modernas técnicas tribológicas (ultramicrodureza, scrascth tests, resistencia al desgaste...) y espectroscópicas óptica por descarga luminiscente (GDOES).

Los resultados obtenidos muestran que las técnicas de PVD son una interesante vía para la obtención de recubrimientos decorativos, de excelente resistencia tanto mecánica como química, y que los métodos de caracterización empleados son adecuados para el control de calidad industrial.
\end{abstract}

Palabras clave: PVD, cerámicas decorativas, tribología, GDOES

\section{Decorative Coatings by PVD on ceramics.}

During the last years PVD coatings has been used for decorative purposes on ceramics and other substrates. Standards layers like TiN, $\mathrm{CrN}, \mathrm{TiCN}$, etc, give us the possibly of obtaining a wide range of colours, with a good mechanical, chemical properties.

This paper gathers the works carried out in the Center of Advance Surface engineering of AIN during the three last years in the field of the decorative coatings. Different layer have been deposited by means of a METAPLAS 232 PVD equipment. A complete chemical and mechanical characterisation have been carried out on the different deposited layers like ultramicroindentation, scratch tests, wear resistance and GDOES.

Keywords: PVD, Decorative coatings, Tribology, GDOES

\section{INTRODUCCIÓN}

Las técnicas de depósitos mediante métodos físicos en fase vapor (PVD) se han consolidado desde hace varios años como una herramienta eficaz para el aumento de las prestaciones de herramientas y componentes empleados en distintos sectores industriales (1-3). Entre las propiedades de este tipo de recubrimientos caben destacarse su elevada resistencia al desgaste, su buena adhesión, su elevada resistencia a la corrosión, etc (1-5).

Además de estas excelentes propiedades físico-químicas, los recubrimientos por PVD ofrecen la posibilidad de obtener una amplia gama de colores, factor que unido a su reducida tasa de producción de residuos ha determinado su introducción en el sector decorativo. En este campo las aplicaciones más extendidas de las técnicas de PVD son en la grifería y los complementos del hogar, elementos habitualmente fabricados en materiales como bronces, latones, zamak, plásticos y cerámicas. Estas nuevas aplicaciones conllevan nuevos requisitos para los tratamientos por PVD, que fundamentalmente se pueden resumir como: la necesidad de obtener un alto grado de reproducibilidad en colores, y la estabilidad tanto mecánica como química de los recubrimientos.

En este trabajo se recogen las experiencias realizadas en el Centro de Ingeniería de Superficies de AIN en la aplicación, y caracterización de tratamientos PVD sobre vidrios y cerámicas decorativas.

\section{EXPERIMENTAL}

\section{Preparación de las muestras}

Dada la alta capacidad de absorción de agua de los vidrios y las cerámicas, la etapa de limpieza superficial previa a la carga del sistema de PVD es uno de los aspectos críticos del proceso. Las distintas muestras fueron sometidas a varias etapas de limpieza: ultrasonidos, lavado en medio básico, aclarado con agua desionizada y secado en estufa durante media hora. 
Los substratos utilizados para los diferentes ensayos son cerámicas arcillosas sobre las que se han aplicado una capa de esmalte.

\section{Tratamiento de las muestras}

Tras las etapas de limpieza, los recubrimientos fueron realizados mediante arco eléctrico reactivo (CVAE) en un equipo METAPLAS MZR323. Este equipo dispone de seis evaporadores que trabajan a $30 \mathrm{~V}$ y $100 \mathrm{~A}$ con un campo magnético débil. Antes del procesos de deposición se llevó a cabo una limpieza iónica (AEGD-Arc Enhaced Glow Discharge) bombardeando el sustrato con argón para eliminar la contaminación superficial. El tiempo de depósito osciló entre los 2 y los 15 minutos, dependiendo del experimento. Este sistema disminuye en gran medida la aparición de las microgotas típicas de los procesos de arco eléctrico $(6,7)$. Los recubrimientos se llevaron a cabo a una temperatura aproximada de $250^{\circ} \mathrm{C}$ empleando nitrógeno como gas reactivo. Los niveles de vacío fueron de $10^{-6}$ mbares de presión base y $10^{-3}$ mbares de presión de trabajo en atmósfera de nitrógeno y argon.

\section{Caracterización de las muestras}

El espesor y la composición química de las muestras recubiertas se estudió empleando técnicas de espectrometría por descarga luminiscente (GD-OES). El equipo empleado fue un JY10000 RF, que dispone de fuente de excitación de radiofrecuencia, y un policromador de 1 metro de distancia focal. Este equipo permite determinar los perfiles de composición química en profundidad, para muestras conductoras y aislantes.

La dureza Universal H.U. (dureza bajo carga con indentador Vickers) de los materiales recubiertos y de las muestra de referencia se han medido con un Microdurómetro FICHERSCOPE H100 empleando una carga final de 5 $\mathrm{mN}(8,9)$. El equipo permite además calcular algunas las propiedades elástico-plásticas superficiales más interesantes como el Módulo de Elasticidad, y el porcentaje de recuperación elástica. Los ensayos de Adhesión se han realizado utilizando un Scracth Tests CSM Revetests, mediante una punta de diamante de tipo Rockwell con un diámetro de contacto de 200 micras, que permite realizar ensayos con cargas de hasta $200 \mathrm{~N}$.

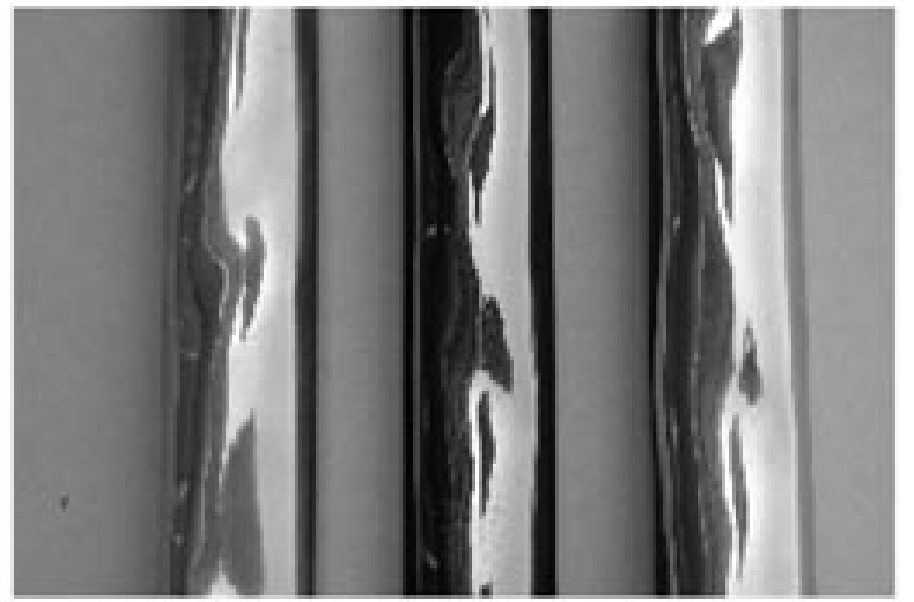

\section{RESULTADOS}

La figuras 1 a-b muestras una fotografía de cerámicas recubiertas con TiN y CrN (Fig. 1a) así como algunas muestras cerámicas sin recubrir (parte izquierda de la Fig 1b). Las distintas muestras recubiertas mostraron características similares de reproducibilidad, en términos de colores obtenidos y grado de homogeneidad de la capa a lo largo del recubrimiento. Este hecho pone de manifiesto la importancia del proceso de eliminación de gases absorbidos en superficie y limpieza iónica previa al comienzo del proceso de recubrimiento. Se constató que los resultados más representativos por su grado de reproducibilidad se obtienen cuando se realizan ciclos de calentamiento previo de los substratos a temperaturas de $300^{\circ} \mathrm{C}, 100^{\circ} \mathrm{C}$ por encima de la temperatura de depósito.

La figura 2 muestra un perfil de concentración atómica de un recubrimiento PVD de TiN de 0.5 micras de espesor. El valor del espesor se obtuvo mediante un procedimiento indirecto de medición del perfil de la huella dejada por el ensayo GD. El perfil de concentración indica que la capa PVD se compone de dos partes atendiendo a la concentración de nitrógeno que presentan. La primera capa de intercara con el substrato presenta una composición de nitrógeno menor que la capa más superficial. Este diseño permite aumentar la adherencia de la capa al substrato.

En la figura 3a se pueden observar las curvas de cargadescarga de la indentación a $5 \mathrm{mN}$ de carga final para las muestras cerámicas recubiertas con TiN y con $\mathrm{CrN}$. Los resultados de ultramicrodureza a $5 \mathrm{mN}$ de carga final muestran que los recubrimientos de $\mathrm{TiN}$ y $\mathrm{CrN}$ poseen durezas del orden de los 11.000-12.000 N/ mm² más del doble del sustrato 4.500 $\mathrm{N} / \mathrm{mm}^{2}$, y superior en un factor 1.5 a los niveles de dureza de recubrimientos tipo cromo duro medidos en el laboratorio del centro, $8.200 \mathrm{~N} / \mathrm{mm}^{2}$. Cabe mencionar que los valores de dureza universal medidos en estos recubrimientos están influenciados por la contribución procedente del substrato, debido al bajo espesor de dichas capas. En efecto, a $5 \mathrm{mN}$ de carga máxima, la profundidad de penetración del indentador es de 0.1 micras. Dicha profundidad es superior al $10 \%$ del espesor de la capa, por lo que la medida no corresponde al valor de dureza real de la capa. De hecho, los valores de dureza universal para el TiN y $\mathrm{CrN}$ reportados en la literatura son de entre $14.000-16.000 \mathrm{~N} / \mathrm{mm}^{2}$ para TiN y de $12.000-13.000$ $\mathrm{N} / \mathrm{mm}^{2}$ para $\mathrm{CrN}$, (dependiendo del método de depósito)

Fig. 1.- TFotografías de diferentes cerámicas recubiertas con capas PVD. A la izquierda se muestran barras cerámicas recubiertas con diferentes capas PVD. A la derecha se muestran otros tipos de capas PVD así como dos barras cerámicas sin recubrir (parte izquierda) 


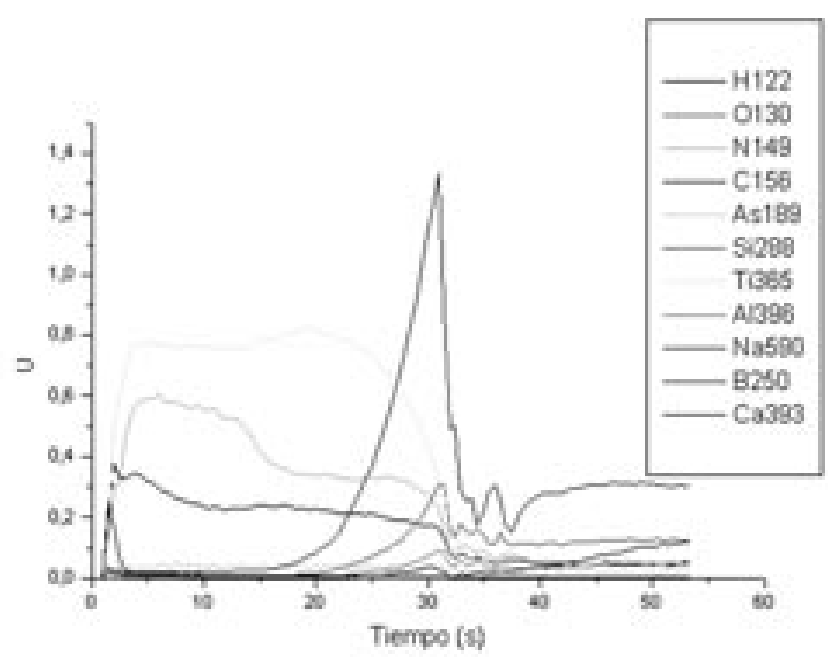

Fig. 2.- Perfil de concentración GD-OES de un recubrimiento PVD de TiN sobre una cerámica esmaltada.
$(10,11)$. En la Fig. 3b se observar el perfil de dureza frente a profundidad para las muestras cerámicas recubiertas con TiN y CrN.

En la figura 4a-c puede observarse un ejemplo de ensayos de adhesión sobre una muestra de TiN. Los ensayos de adherencia realizados sobre los recubrimientos TiN y $\mathrm{CrN}$ depositados sobre las muestras cerámicas revelaron cargas críticas de fallo de capa de aproximadamente 14-15 N. Esto se observa con más detalle en la Fig. 4a donde se ha representado la emisión acústica producida durante el ensayo. En esta figura la emisión acústica (eje vertical derecho) aumenta de forma abrupta cuando la carga aplicada sobrepasa los 15 N. A partir de estos valores de carga, se producen diferentes tipos de fallos cohesivos y adhesivos que determinan la resistencia mecánica de la capa. El las figuras $4 \mathrm{~b}-\mathrm{c}$ se muestran imágenes mediante microscopía óptica de los fallos característicos observados en el ensayo Scratch de la Fig. 4a. Estas imágenes muestran el tipo de fallo que se producen en condiciones de carga aplicada. Entre otros tipos de fallos producidos,

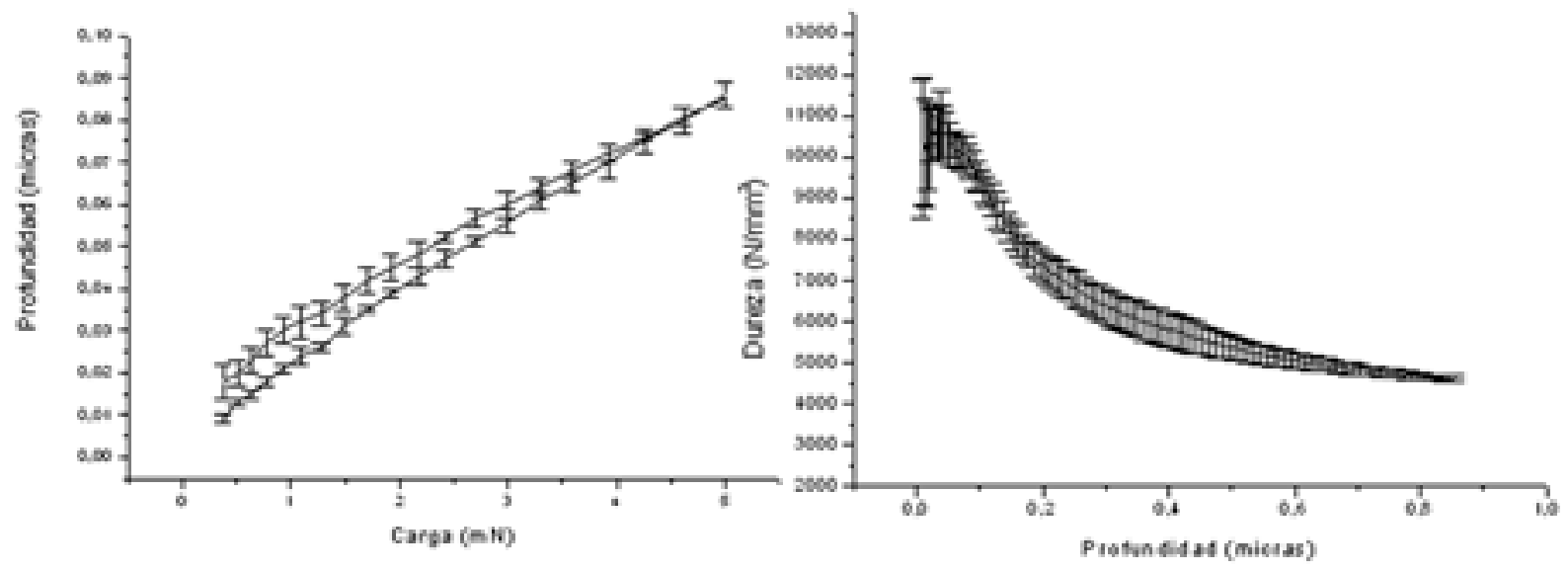

Fig. 3.- Ensayos de microdureza sobre una capa PVD de TiN sobre una cerámica esmaltada. (a) ensayo de indentación con una carga máxima de $5 \mathrm{mN}$. (b) Perfil de dureza universal en función de la profundidad de indentación.

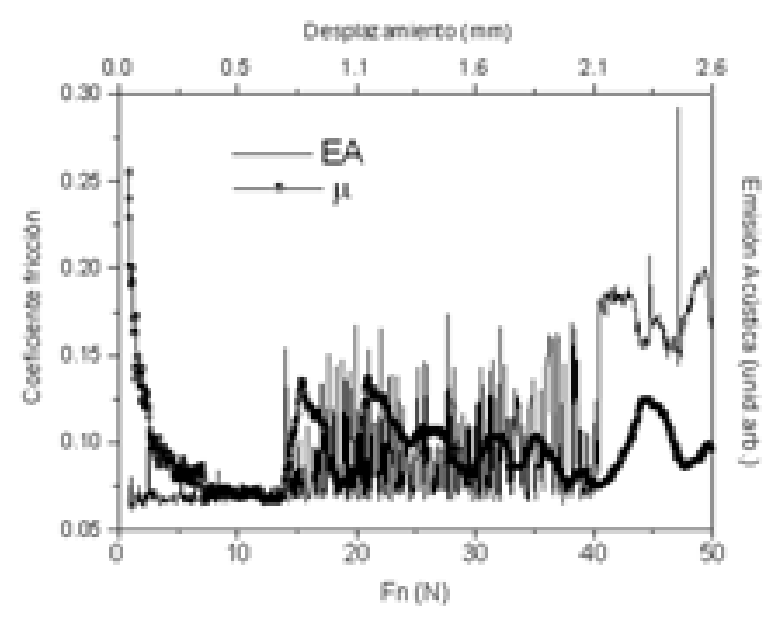

se observan fracturas que se han originado en la huella del arañazo y se han propagado hacia fuera (Fig. .4b). A mayores cargas, se observan partes de la capa que se han despegado del substrato.

Cabe destacar que, debido a la prácticamente nula existencia de otros trabajos que reporten este tipo de ensayos, sobre TiN/CrN depositado en cerámicas, estos resultados no nos permiten establecer de forma absoluta el grado de adhesión, pero si apuntar que estos valores pueden considerarse muy buenos en comparación con otras técnicas de recubrimiento como los recubrimientos electrolíticos y galvánicos.

\section{CONCLUSIONES}

Mediante técnicas de PVD por arco eléctrico se han depositado recubrimientos en forma de lámina delgada sobre cerámicas, con fines decorativos. Los resultados de los ensayos realizados indican que:

Es posible obtener un alto grado de reproducibilidad si se controlan adecuadamente los parámetros del proceso. 

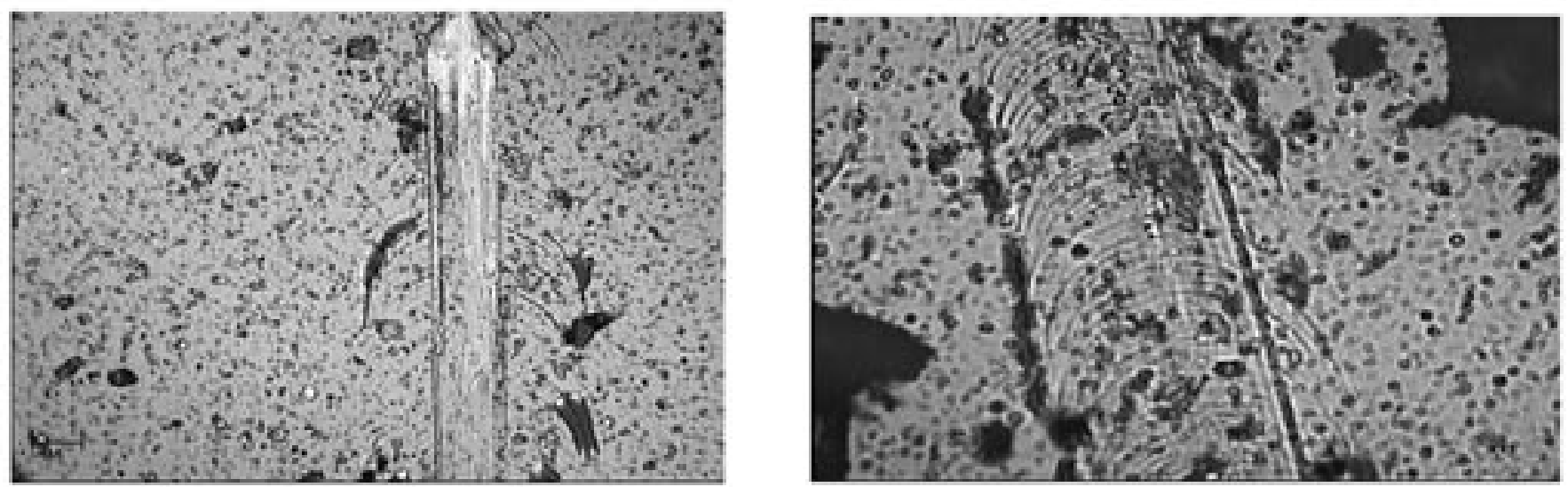

Fig. 4.- b-c. Ensayos Scratch sobre TiN crecido en cerámicos. (a) diagrama de emisión acústica. (b) Imágenes de las huellas de producidas tras el proceso de Scratch.

Entre estos parámetros, la limpieza previa, y los ciclos de calentamiento y limpieza iónica, juegan un papel fundamental.

Se depositaron recubrimientos de entre $50 \mathrm{~nm}$ y $500 \mathrm{~nm}$ de espesor. La microdureza de estos recubrimientos es más de un $120 \%$ mayor que la del sustrato, y más del doble de otros tipos de recubrimientos como los recubrimientos electrolíticos o galvánicos.

A pesar de no existir otros resultados comparativos con los valores de adhesión obtenidos, parece apuntarse un alto grado de adhesión entre la capa y el sustrato.

Los resultados obtenidos indican que las técnicas por PVD arco eléctrico, son una herramienta sumamente eficaz para recubrir cerámicas y materiales aislantes con fines decorativos, pero además estos recubrimientos ofrecen una protección muy superior a la de otros métodos de recubrimientos tradicionales.

\section{AGRADECIMIENTOS}

Los autores desean expresar su agradecimiento al programa INERREG IIIB del marco espacio atlántico por su apoyo a través del proyecto PLASMANTET E26 y a la cofinanciación del Gobierno de Navarra.

\section{BIBLIOGRAFÍA}

1. R. J. Rodríguez, J. A. García, A. Medrano, R. Sánchez, M. Rico, R. Martínez, C. Labrugere, M. Lahaye, and A. Guette, Tribological behaviour of hard coatings deposited by arc evaporation PVD, Vacuum 67, 559 (2002).

2. E. Román, J.L. de Segovia, A. Alberdi, J. Calvo, J. Laucirica Tribological behaviour of hard coatings deposited by arc, Vacuum, 43, 685-687 (1992).

3. E. Román, J.L. de Segovia, A. Alberdi, J. Calvo, Laucirica, Comparative study of the interface composition of TiN and TiCN hard coatings on high speed steel substrates obtained by arc discharge, J. Mater Sci Eng,; A163, 197-200 (1993)

4. Guu YY, Lin JF, Ai CF, The Tribological Characteristics of Titanium Nitride, Thin solid films 302 193-200 (1997).

5. Byeli AV, Minevich AA, Stepanenko AV, Gick LA, Kholodilov OV, Wear resistance and structure of (Ti, Al)N coatings, J Phys D: Appl Phys, 25, 292 296 (1992).

6. J. Vetter, Vacuum arc coatings for tools:potencial and applications, Surf. Coat. Technol, 76-77 719-724 (1995).

7. Farhat ZN, Ding Y, Northwood DO,Effect of Grain Size on Fricction and Wear of Nanocrystaline Aluminum, Alpas AT. Mater Sci Eng 206, 302-308 (1996).

8. Loubet JL, Georges JM, Meille G, Microindentation Techniques in materials Science an Engineering, ASTM STP, 889, 72-89 (1986).

9. Oliver WC, Pharr GM, An improved technique for determining hardness and elastic madulus using load and displacement, J Mater Res, 7, 1564-1583 (1992).

10. S.T. Oyama in The Chemistry of Transition Metal Carbides and Nitrides, Edited by S.T. Oyama. Blackie Academic and Professional Glasgow UK (1996).

11. G. Wei, A. Rar, J.A. Barnard, Composition, structure and nanomechanical properties of DL-sputtered $\mathrm{CrN}_{\mathrm{x}}(0 \leq \mathrm{x} \leq 1)$ thin films, Thin Solid, Films, 460, 398-399 (2001).

Recibido: 01.12 .03

Aceptado: 19.04 .05 